\title{
Identification and functional analysis of changes to the ox-LDL-induced microRNA-124-3p/DLX5 axis in vascular smooth muscle cells
}

\author{
Chunwen Jia ${ }^{A-D}$, Feng Gao ${ }^{A, C-F}$, Yanan Zhao $0^{B, C}$, Siyang Ji ${ }^{C}, D$, Shidao Cai ${ }^{B, D}$ \\ Department of Cardiology, Zhongshan Hospital affiliated to Xiamen University, China \\ A - research concept and design; $\mathrm{B}$ - collection and/or assembly of data; $\mathrm{C}$ - data analysis and interpretation; \\ $D$ - writing the article; $E$ - critical revision of the article; $F$ - final approval of the article
}

Address for correspondence

Feng Gao

E-mail:fenggao_xmhos@163.com

Funding sources

The study was supported by departmental funds provided by Department of Cardiology, Zhongshan Hospital, affiliated to Xiamen University (grant No. ZSYY-XN-res-201806).

Conflict of interest None declared

Received on March 25, 2021

Reviewed on August 17, 2021

Accepted on August 25, 2021

Published online on October 27, 2021

Cite as

Jia C, Gao F, Zhao Y, Ji S, Cai S. Identification and functional analysis of changes to the ox-LDL-induced microRNA124-3p/DLX5 axis in vascular smooth muscle cells. Adv Clin Exp Med. 2021;30(12):1271-1281.

doi:10.17219/acem/141580

DOI

10.17219/acem/141580

Copyright

Copyright by Author(s)

This is an article distributed under the terms of the

Creative Commons Attribution 3.0 Unported (CC BY 3.0)

(https://creativecommons.org/licenses/by/3.0/)

\begin{abstract}
Background. The microRNA (miR)-mRNA axes involved in oxidized low-density lipoprotein (ox-LDL)induced vascular smooth muscle cells (VSMCS) proliferation/apoptosis imbalance need to be investigated in more detail.

Objectives. To investigate the function and any relevant changes to miR-mRNA axes in the ox-LDL-induced proliferation/apoptosis imbalance in VSMCS.

Materials and methods. Human VSMCs were cultured and treated with ox-LDL in vitro. The differentially expressed (DE) miRs and mRNAs in VSMCs following 48-h ox-LDL treatment were detected using RNA sequencing. Candidate miRs and mRNAs were further selected, based on comprehensive bioinformatics analysis. Changes in the expression of candidate miRs and mRNAs in ox-LDL-treated VSMCs were confirmed with quantitative real-time polymerase chain reaction (RT-qPCR) and western blot. The inhibitory effect of candidate miRs on mRNAs were demonstrated using a dual luciferase reporter assay. The functional role of candidate miRs and mRNAs on proliferation, cell cycle and apoptosis of VSMCs were estimated using cell couting kit-8 (CCK-8) and flow cytometry assays, respectively.
\end{abstract}

Results. The RNA sequencing data indicated that 577 mRNAs and 81 miRs were significantly upregulated in VSMCs following ox-LDL treatment. Gene function and pathway enrichment analysis suggested that the DEmRNAs and miRs participate in the regulation of proliferation, cell cycle and apoptosis. Increased expression of DLX5 with decreasing miR-124-3p levels were confirmed in ox-LDL-treated VSMCS. The miR-124-3p could inhibit the DLX5 level by binding the 3' UTR of DLX5 mRNA. Functional analysis showed that the alteration of miR-124-3p/DLX5 expression mediated the effect of ox-LDL on VSMCs proliferation/apoptosis imbalance.

Conclusions. The ox-LDL affects the VSMC proliferation/apoptosis balance via the miR-124-3p/DLX5 axis, which results in VSMC hyperproliferation. The miR-124-3p/DLX5 axis might serve as a therapeutic molecular target to reverse the effect of ox-LDL and prevent atherosclerosis (AS) development and progression.

Key words: atherosclerosis, microRNA, vascular smooth muscle cell, oxidized low-density lipoprotein, DLX5 


\section{Background}

Atherosclerosis (AS) is a vascular disease characterized by stiff, thickened artery walls with reduced elasticity, together with the formation of atherosclerotic plaques within the intimal layer. ${ }^{1}$ Its development and progression involves vascular endothelial cell damage and dysfunction, inflammatory cell recruitment, lipid deposition, and thrombosis, in addition to abnormal vascular smooth muscle cell (VSMC) proliferation. ${ }^{2}$ Excessive proliferation of VSMCs causes an inflammatory response and thickening of vessel walls, both of which facilitate lipoprotein deposition and fibrous cap formation. ${ }^{3}$

Elevated low-density lipoprotein (LDL) is a major risk factor for AS. Low-density lipoprotein comprises a core (mainly triglycerides and cholesterol lipids) and a surface rich in polyunsaturated fatty acids. ${ }^{4}$ Therefore, LDL is easily oxidized and modified by stimulatory factors such as cigarette smoke, hyperglycemia and diabetes. ${ }^{5}$ Oxidized LDL (ox-LDL) is even more potent in inducing AS. It is able to damage vascular endothelial cells, causing dysfunction and increased permeability, which initiates AS. ${ }^{6}$ Furthermore, ox-LDL intake upregulates the transition of macrophages to foam cells within plaques. Previous studies have reported that ox-LDL promotes excessive proliferation of VSMCs by promoting endothelin-1 secretion and inhibiting prostacyclin synthesis. ${ }^{7}$ Some of the ox-LDLinduced cytokines involved in the regulation of VSMC proliferation include platelet-derived growth factor (PDGF), basic fibroblast growth factor (bFGF) and transforming growth factor beta (TGF- $\beta$ ). ${ }^{8}$ Reversing ox-LDL-induced VSMC hyperproliferation may represent a new strategy in AS treatment. However, the mechanisms underlying oxLDL-induced regulation of VSMC proliferation need to be explored prior to the clinical application of this strategy. ${ }^{9}$

Recent studies investigating the regulatory mechanisms of microRNAs (miRs) have shown that miRs play an important regulatory role in ox-LDL-induced VSMC proliferation, and several potential miR therapeutic targets have been identified there. Sun et al. reported that miR-490-3p is associated with increased ox-LDL-induced VSMC proliferation through the regulation of its target gene, $P A P P-A$. The Ox-LDL inhibits miR-490-3p expression, and the upregulated miR-490-3p reduces ox-LDL-induced abnormal VSMC proliferation by inhibiting $P A P P-A .{ }^{10}$ Furthermore, Wang et al. identified another ox-LDL-induced miR, miR-195. They found that ox-LDL inhibits miR-195 levels in VSMCs and that miR-195 inhibits VSMC proliferation and migration. ${ }^{11}$

\section{Objectives}

In this study, we aimed to analyze RNA sequencing data from ox-LDL-treated VSMCs to find novel miR-messenger RNA (mRNA) axes involved in ox-LDL-induced VSMC proliferation. We selected differentially expressed (DE) miRs and mRNAs in ox-LDL-treated cells and conducted functional and bioinformatics analyses on these genes. We selected the miR-124-3p/DLX5 axis as one potentially related to VSMC proliferation, which was confirmed following ox-LDL treatment. Considering that ox-LDL promotes VSMC proliferation by breaking the cell proliferation/apoptosis balance, we further explored the regulatory function of the miR-124-3p/DLX5 axis in VSMC proliferation and apoptosis.

\section{Materials and methods}

\section{Cell culture and ox-LDL treatment}

Human aorta smooth muscle cells (HA-VSMC, product code: CRL-1999) were obtained from the American Type Culture Collection (ATCC, Manassas, USA) and cultured using F-12K media (Thermo Fisher Scientific, Waltham, USA) in a humidified incubator at $5 \%$ $\mathrm{CO}_{2}$ and $37^{\circ} \mathrm{C}$. To make the complete growth medium, the following components were provided by Procell Life Science\&Technology (Wuhan, China) and added to the base medium: $0.05 \mathrm{mg} / \mathrm{mL}$ of ascorbic acid; $0.01 \mathrm{mg} / \mathrm{mL}$ of insulin; $0.01 \mathrm{mg} / \mathrm{mL}$ of transferrin; $10 \mathrm{ng} /$ $\mathrm{mL}$ of sodium selenite; $0.03 \mathrm{mg} / \mathrm{mL}$ of Endothelial Cell Growth Supplement (ECGS); fetal bovine serum (FBS; Thermo Fisher Scientific) to a final concentration of $10 \%$, HEPES to a final concentration of $10 \mathrm{mM}$, and TES to a final concentration of $10 \mathrm{mM}$. Antibiotics were not added into the medium. The HA-VSMCs in their $5^{\text {th }}$ passage were treated with $100 \mu \mathrm{g} / \mathrm{mL}$ of ox-LDL (Thermo Fisher Scientific) for $24 \mathrm{~h}$ before further experiments.

No approval was required from bioethical committee due to the fact that no human or animals were enrolled in the present study.

\section{RNA sequencing and data analysis}

After $48 \mathrm{~h}$ of exposure to ox-LDL, cells were collected, and samples sent for RNA sequencing (BGI Technology Service, Shenzheng, China). The DE-mRNAs and miRs were analyzed using theDESeq2 package in $\mathrm{R}$ software v. 3.4.1 (R Foundation for Statistical Computing, Vienna, Austria). The DE-genes are defined as genes with a foldchange of expression $>2.0$ and $\mathrm{p}<0.05$.

\section{Bioinformatic analysis}

To explore the potential biological function of DE mRNA and miRs, further functional and signaling pathway analyses were performed. Gene Ontology (GO) gene enrichment analysis and Kyoto Encyclopedia of Genes and Genomes (KEGG) signaling pathway enrichment analyses were performed on a set of DE-mRNAs using 
data from the Database for Annotation, Visualization and Integrated Discovery (DAVID; https://david.ncifcrf.gov/). Signaling pathway analyses for DE-miRs were conducted using the online tool mirPath v. 3 (http://snf-515788. vm.okeanos.grnet.gr/). The miR-mRNA target relationship was predicted using miRanda-mirSVR tools (http:// www.microrna.org/microrna/getMirnaForm.do).

\section{Quantitative real-time polymerase chain reaction}

The total RNA, including miRs was isolated from cells using TRIzol reagent (Thermo Fisher Scientific). To evaluate miR-124-3p expression levels, RNA samples were reverse transcribed into cDNA using miScript Reverse Transcription Kit (Qiagen, Shanghai, China) and amplified using miScript SYBR-Green PCR Kits (cat. No. 218073; Qiagen). The expression value of miR-124-3p was normalized to the reference gene $R N U 6 B$. The primers of miR-124-3p and RNU6B were provided using miScript Primer Assay Kit (cat. No. 218300; Qiagen) and are patent-protected. To detect the level of DLX5, cDNA was synthesized using PrimeScript RT reagent Kit (cat. no. RR037A; TaKaRa, Beijing, China) and analyzed using TB Green ${ }^{\circledR}$ Premix Ex Taq ${ }^{\mathrm{TM}}$ II Kit (cat. No. RR820A; TaKaRa). The expression value of $D L X 5$ was normalized to GAPDH. The forward primer of DLX5 was 5'TTCCAAGCTCCGTTCCAGAC-3'. The reverse primer of DLX5 was 5'-GAATCGGTAGCTGAAGACTCG-3'. The forward primer of GAPDH was 5'-GGAGCGAGATCCCTCCAAAAT-3'. The reverse primer of GAPDH was 5'-GGCTGTTGTCATACTTCTCATGG-3'.

\section{Western blot}

Treated cells were lysed on ice in radioimmunoprecipitation assay (RIPA) buffer supplemented with a protease and phosphatase inhibitor cocktail (Beyotime, Shanghai, China). The concentration of extracted protein was measured using BCA protein assay kit (Beyotime). Equal amounts of protein samples were loaded and separated with $10 \%$ polyacrylamide gel electrophoresis (PAGE) gel. After electrophoresis, samples were transferred to nitrocellulose membranes and membranes were blocked using $5 \%$ fat-free milk. Then, membranes were incubated with rabbit monoclonal primary antibodies of DLX5 (Abcam, Cambridge, UK) and GAPDH (Abcam). Next, goat antirabbit IgG H\&L (horseradish peroxidase (HRP)-labeled) secondary antibody (Abcam) was used. Protein bands were detected using the electrochemiluminescence (ECL) detection kit (Beyotime).

\section{Cell transfection}

The pcDNA3.1-DLX5 expression vectors were constructed and transfected into HA-VSMC to overexpress
DLX5 expression. An empty pcDNA3.1 vector served as the transfection negative control (NC). To knockdown $D L X 5$ expression, siRNA-DLX 5 were transfected into cells. To up- or downregulate the expression of miR-124-3p, specific miR-mimics and miR-inhibitors (Qiagen) were used. For siRNA and miR-mimics, the Allstars sequence (cat. No. 1027280; Qiagen) was used as the transfection NC. For miR-inhibitors, miScript Inhibitor Negative Control (cat. No. 1027272; Qiagen) was used as the transfection NC. Lipofectamine 2000 Reagent was used 48 times according to the manufacturer's instructions. Once the transfection was confirmed with qRT-PCR (Fig. 1), cells were used for further experiments.
A

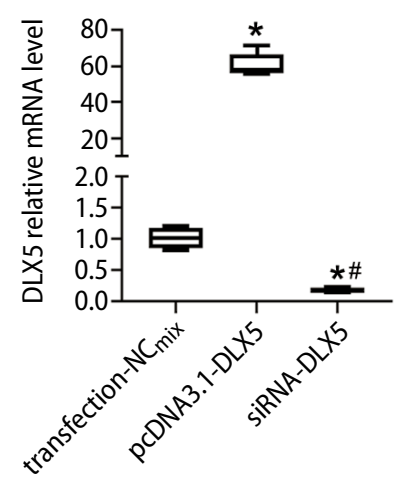

B

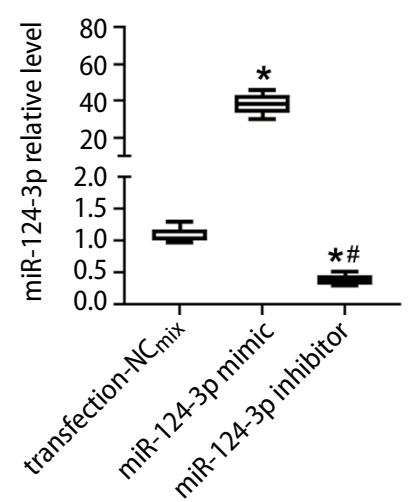

Fig. 1. Transfection effects of human aorta vascular smooth muscle cells (HA-VSMCs) were confirmed using quantitative real-time polymerase chain reaction (RT-qPCR). A. The effect of pcDNA3.1-DLX5 and siRNA-DLX5; $B$. The effect of miR-124-3p mimic and miR-124-3p inhibitor

* $p<0.05$ compared with matched transfection negative control (NC) mixture; ${ }^{*} p<0.05$ compared with pcDNA3.1-DLX5 or miR-124$3 p$ mimic. Transfection $\mathrm{NC}_{\text {mix }}$ was a mixture of Allstars sequence and empty pcDNA3.1 in $A(n=5)$. Transfection $N C_{\text {mix }}$ was a mixture of Allstars sequence and miScript Inhibitor Negative Control in B $(n=5)$.

\section{Proliferation analysis}

Treated cells were plated into 96-well plates at 5000 cells/ well. At $0 \mathrm{~h}, 24 \mathrm{~h}, 48 \mathrm{~h}$ and $72 \mathrm{~h}$ timepoints, $10 \mu \mathrm{L}$ Cell Counting Kit- 8 reagent (Beyotime) was added into each well $(200 \mu \mathrm{L}$ medium/well) and the cells were incubated at $37^{\circ} \mathrm{C}$ for $1 \mathrm{~h}$. The absorbance was measured at $450 \mathrm{~nm}$ using an automatic microplate reader BioTek Elx800 (BioTek, Winooski, USA) and normalized to the blank well. Relative proliferation $=$ normalized absorbance value at other timepoints $/ \mathrm{N}=$ normalized absorbance value at $0 \mathrm{~h}$.

\section{Flow cytometry analysis for cell cycle}

Treated cells were collected and washed with cold phosphate-buffered saline (PBS). Cell cycle assay was performed using BD Pharmingen BrdU Flow Kit (Becton Dickinson Biosciences, San Diego, USA). In brief, cells were labeled with and fixed/permeabilized using BD Cytofix/Cytoperm Buffer (Becton Dickinson Biosciences). Then, cells were 
treated with deoxyribonuclease (DNase) and incubated with fluorescein isothiocyanate (FITC)-conjugated anti-BrdU. After staining with 7-AAD and resuspending, Guava ${ }^{\circledR}$ easyCyte 8HT Benchtop Flow Cytometer (Merck Millipore, Darmstadt, Germany) was used to perform flow cytometry analysis. Data were analyzed using Guava ${ }^{\circledR}$ InCyte and GuavaSuite software v. 3.0 (Merck Millipore).

\section{Apoptosis assay}

Cells were plated in 96-well plates and treated with indicated transfection contents and/or ox-LDL. Following $24 \mathrm{~h}$ of treatment, cells were incubated with prepared $2 \times$ detection reagent from the RealTime-Glo ${ }^{\mathrm{TM}}$ Annexin V Apoptosis Assay Kit (Thermo Fisher Scientific). Luminescence was detected using Spark ${ }^{\circledR} 10 \mathrm{M}$ fluorescence plate reader (Tecan, Männedorf, Switzerland) to indicate apoptosis.

\section{Dual-luciferase reporter assay}

The wild-type and mutant DLX5 3'-UTR fragment was subcloned into the pMIR REPORT ${ }^{\mathrm{TM}}$ miRNA expression firefly luciferase reporter vectors (Ambion, Austin, USA). The pRL-TK reporter control vectors (Ambion) expressing Renilla luciferase were used as the internal control. Luciferase reporter vector (DLX5 3'-UTR-WT and $D L X 5$ 3'-UTR-MUT) or control vectors were co-transfected with miR-124-3p mimic or mimic controls, using Lipofectamine 2000 according to the manufacturer's protocols. After $24 \mathrm{~h}$ of co-transfection, firefly luciferase activity was determined using the Dual-Luciferase ${ }^{\circledR}$ Reporter Assay System (Promega, Beijing, China), and normalized to the background Renilla luciferase activity.

\section{Statistical analyses}

Statistical analyses were performed using $\mathrm{R}$ v. 3.4.1 (R Foundation for Statistical Computing, Vienna, Austria). Results were presented as the means \pm standard deviation (SD). Differences in means between 2 groups were determined using Student's t-test. For groups of more than 2, a one-way analysis of variance (ANOVA) with a Bonferroni's post hoc test or Dunnett's multiple comparisons test was performed to determine significance. A p-value of $<0.05$ was considered statistically significant.

\section{Results}

\section{Changes in mRNA and miR expression profiles and functional analysis in ox-LDL-treated VSMCs}

High-throughput RNA sequencing showed that 577 mRNAs had significantly altered expression in VSMCs,
$24 \mathrm{~h}$ after ox-LDL treatment (fold-change (FC) of expression $>2.0 ; \mathrm{p}<0.05)$, of which 219 genes were upregulated and 258 downregulated (Fig. 2A,C). In addition, a significant change in the expression of $81 \mathrm{miRs}$ following oxLDL treatment was observed (FC $>2.0 ; \mathrm{p}<0.05)$, of which 63 were upregulated and 18 downregulated (Fig. 2B,D). We also performed Gene Ontology (GO) gene enrichment analysis of DE-mRNAs (Fig. 3A). The DE-mRNAs were involved in a range of cellular processes, such as "cell proliferation", "cell growth" and "regulation of cell cycle". Kyoto Encyclopedia of Genes and Genomes (KEGG) signaling pathway enrichment analysis of DE-mRNAs (Fig. 3B) demonstrated that the DE-mRNAs are related to cell cycle and apoptosis pathways. Pathway enrichment analysis of DE-miRs, whose expression was significantly altered, showed that the target genes of these miRs are also involved in apoptosis and cell cycle pathways (Fig. 3C). Taken together, the ox-LDL responsive DE-mRNAs and DE-miRs may play a functional role in the regulation of proliferation, cell cycle and apoptosis in VSMCs.
A

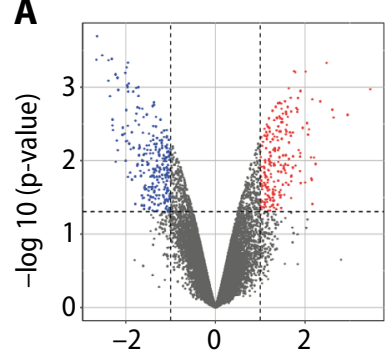

$\log 2$ fold change

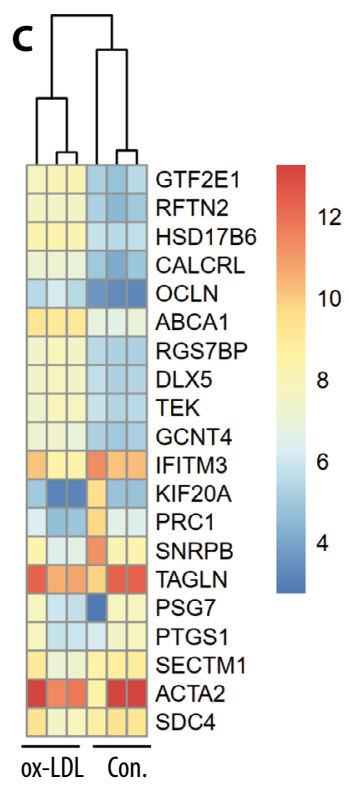

D
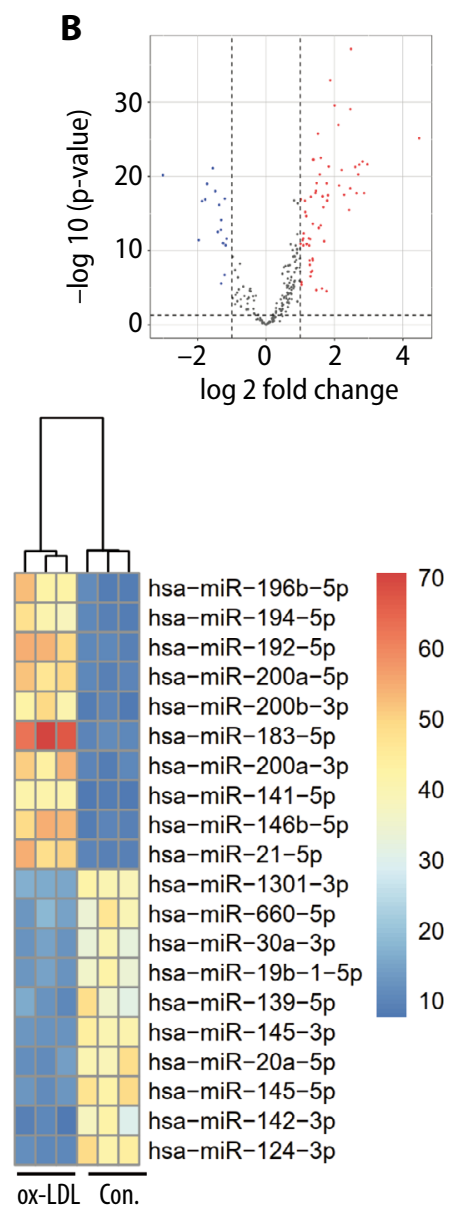

Fig. 2. Differentially expressed mRNAs and microRNAs after $48 \mathrm{~h}$ of oxidized low-density lipoprotein (ox-LDL) treatment. A. Volcano plot showing 219 significantly upregulated and 258 downregulated mRNAs in cells after $48 \mathrm{~h}$ of ox-LDL treatment; $\mathrm{B}$. The volcano plot showing 63 significantly upregulated and 18 downregulated microRNAs in cells after 48 h of ox-LDL treatment; C. Heatmap showing top 10 upregulated and downregulated mRNAs in cells after 48 h of ox-LDL treatment; D. Heatmap showing top 10 upregulated and downregulated mRNAs in cells after $48 \mathrm{~h}$ of ox-LDL treatment 
A

$\mathrm{GO}$ - biological progress

regulation of transcription cexpression cell proliferation rellular response to zinc ion regulation of cell growth negative regulation of stress regulation of cell cycle response to interferon-beta type I interferon signaling pathway

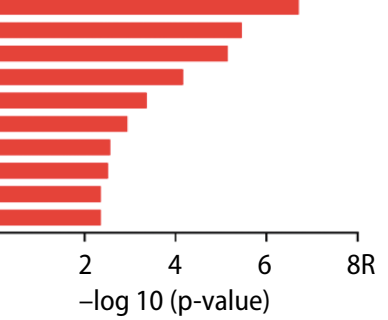

GO - molecular function

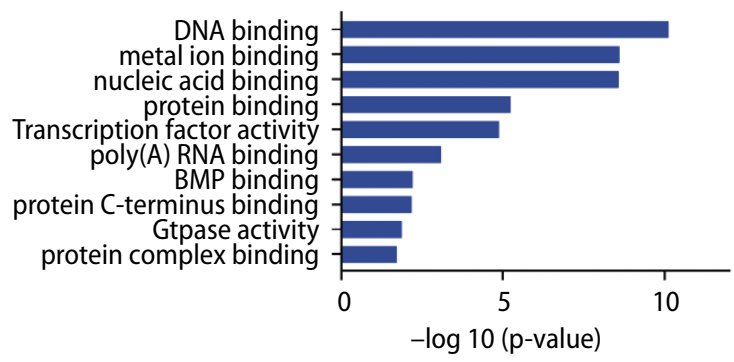

C

GO - cellular component

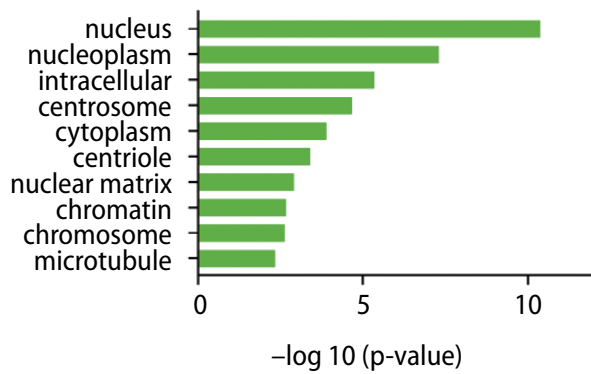

B
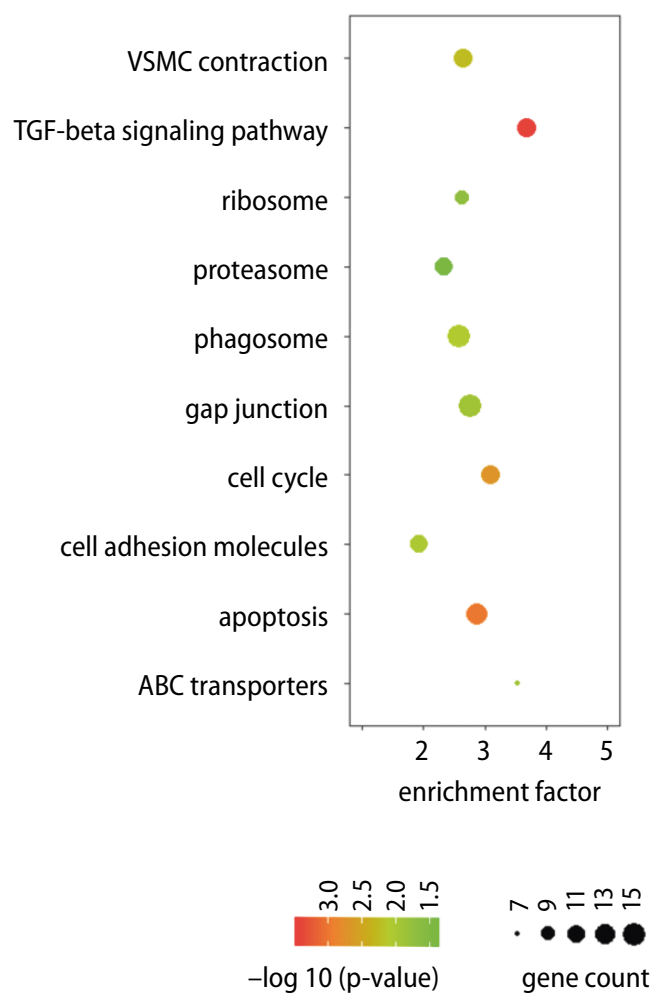

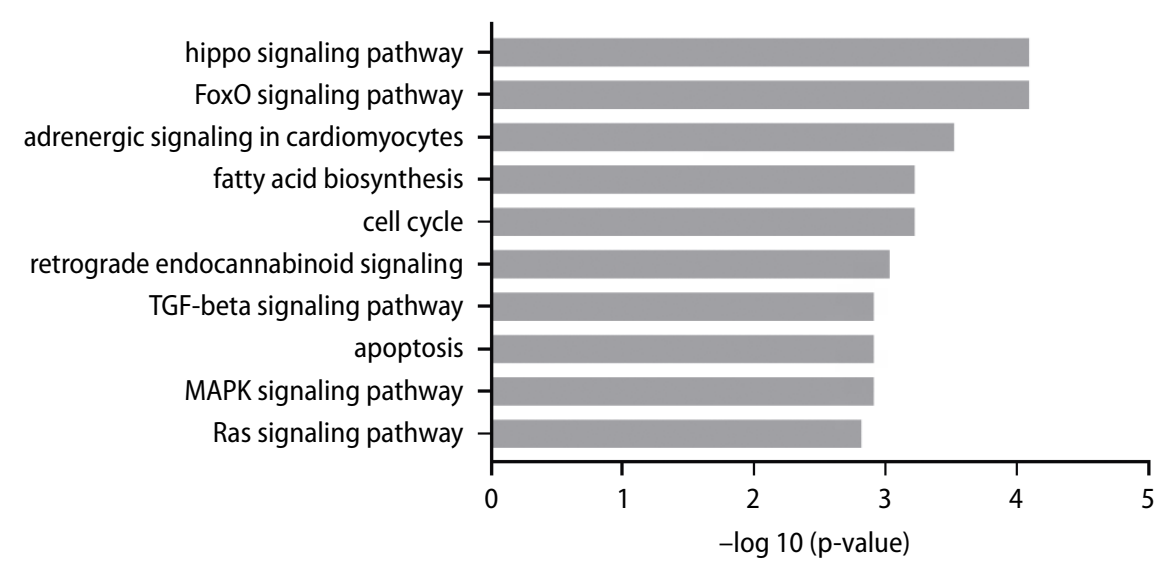

Fig. 3. Functional annotation analysis for differentially expressed mRNAs and microRNAs in human aorta vascular smooth muscle cells (HA-VSMCs) after $48 \mathrm{~h}$ of oxidized low-density lipoprotein (ox-LDL) treatment. A. Top 10 Gene Ontology (GO) gene enrichment analysis results for differentially expressed mRNAs showed their potential biological progress, molecular function and cellular component; B. Top 10 Kyoto Encyclopedia of Genes and Genomes (KEGG) signaling pathway enrichment analysis result for differentially expressed (DE) mRNAs; C. Top 10 pathway enrichment analysis results for DE microRNAs

\section{Aberrant changes in the miR-124-3p/DLX5 axis in ox-LDL-treated VSMCs}

Based on GO gene enrichment analysis and KEGG pathway enrichment analysis results, we selected DEgenes related to the proliferation, apoptosis and cell cycle of VSMCs as candidate genes for further analysis. A total of 35 genes were potentially related to proliferation, apoptosis and cell cycle of VSMCs (Fig. 4A). Among these, $D L X 5$ had the highest upregulation. Next, we predicted miRs that could complementarily bind to DLX5 mRNA 3'UTR using the miRabda-mirSVR tools and compared 
A

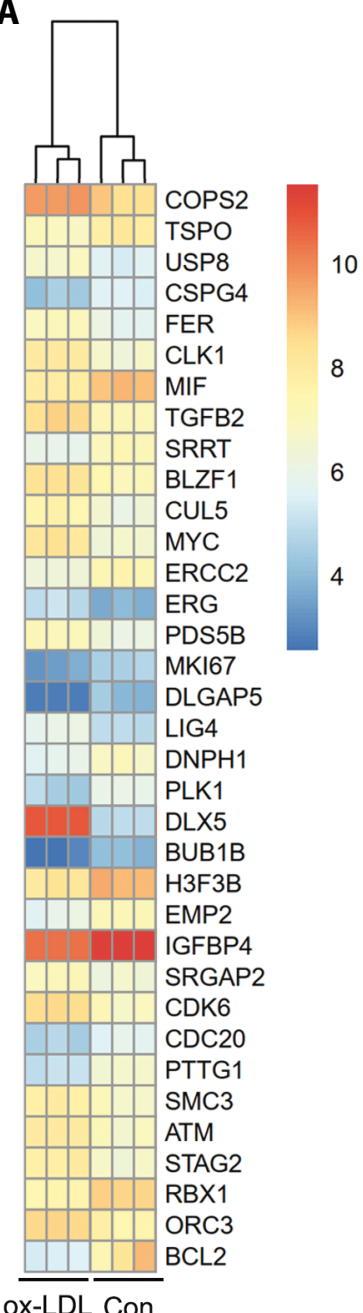

B

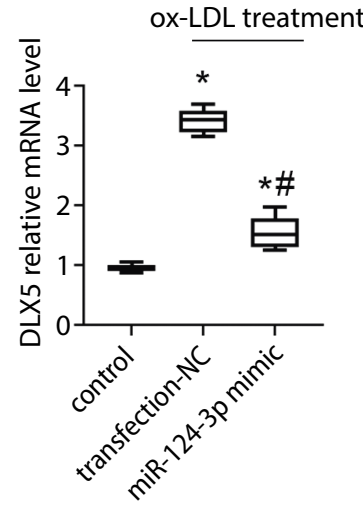

D

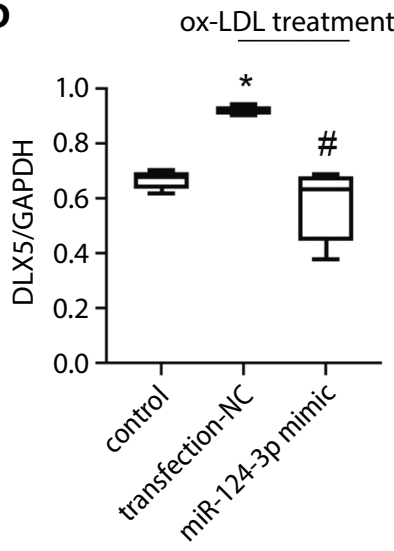

C

$$
\text { DLX5 }
$$

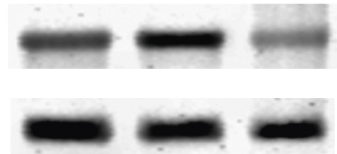

GAPDH

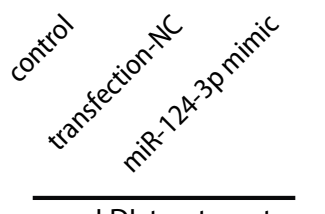

ox-LDL treatment

\section{$\mathbf{E}$}

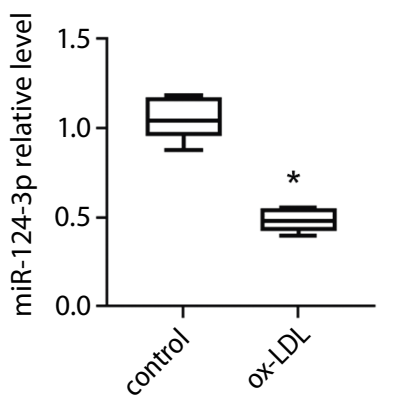

Fig. 4. Oxidized lowdensity lipoprotein (ox$\mathrm{LDL}$ ) increased expression of DLX5 and reduced miR-124-3p level in human aorta vascular smooth muscle cells (HA-VSMCs).

A. Heatmap showing expression alteration of proliferation, cell cycle and apoptosis-related candidate genes induced by ox-LDL in HA-VSMCs; B. Forty-eight hours of oxLDL treatment increased mRNA level of DLX5; $C$ and D. Forty-eight hours of ox-LDL treatment increased protein level of DLX5; E. Forty-eight hours of ox-LDL treatment reduced miR-124-3p level in cells

* $p<0.05$ compared with control; \# $p<0.05$ compared with transfection negative control (NC); $n=5$. these results with the ox-LDL-treated downregulated miR list, finding only miR-124-3p being overlapped. We found that less than $200 \mu \mathrm{g} / \mathrm{mL}$ of ox-LDL induced proliferation and apoptosis at same time, but $200 \mu \mathrm{g} / \mathrm{mL}$ or more inhibited proliferation (Fig. 5A,B). Hence, we selected $100 \mu \mathrm{g} / \mathrm{mL}$ of ox-LDL for further study, which was shown to significantly enhance cell proliferation (Fig. 5C). Furthermore, this dose of ox-LDL also induced significant apoptosis after $12 \mathrm{~h}$ of treatment, although there was no further increase to the apoptotic rate with further treatment (Fig. 5D). We suggest that ox-LDL could promote the proliferation of VSMC and induce apoptosis at the same time, but the pro-proliferative effect of ox-LDL is greater than the pro-apoptotic effect, hence the imbalance.

Following the treatment with $100 \mu \mathrm{g} / \mathrm{mL}$ ox-LDL, the DLX5 expression in VSMCs was detected using qRT-PCR and western blotting. These results showed that ox-LDL treatment significantly upregulates $D L X 5$ at $24 \mathrm{~h}$ (Fig 4B-D). Transfection with the miR-124-3p mimic antagonized the effect of ox-LDL on DLX5. The qRT-PCR verification showed that ox-LDL treatment significantly decreased miR-124-3p expression after $24 \mathrm{~h}$ (Fig. 4E), although the $D L X 5$ mRNA level did not significantly change following ox-LDL treatment for $12 \mathrm{~h}$ (Fig. 5E). Yet, changes of miR-124-3p could be observed after $12 \mathrm{~h}$ of ox-LDL treatment (Fig. 5F). The DLX5 and miR-124-3p did not further change after $48 \mathrm{~h}$ of ox-LDL treatment.

Next, we analyzed the inhibitory effect of miR-124-3p on its molecular target, DLX5. These data showed that mRNA and protein levels of DLX5 significantly decreased in response to miR-124-3p upregulation, and significantly increased after downregulating miR-124-3p in VSMCs $(\mathrm{p}<0.05)$ (Fig. 6A-C). The dual luciferase reporter assay indicated that miR-124-3p directly binds to the DLX5 3'UTR. Overall, these results indicated that ox-LDL can alter the balance of the miR-124-3p/DLX5 axis in VSMCs.

\section{Oxidized low-density lipoprotein-induced VSMC proliferation/apoptosis imbalance via the miR-124-3p/DLX5 axis}

Next, we analyzed the function of the miR-124-3p/DLX5 axis in VSMCs. Overexpressed miR-124-3p inhibited VSMC proliferation $(\mathrm{p}=0.03)$, while VSMC proliferation was increased $(\mathrm{p}=0.04)$ when miR-124-3p was inhibited 
A
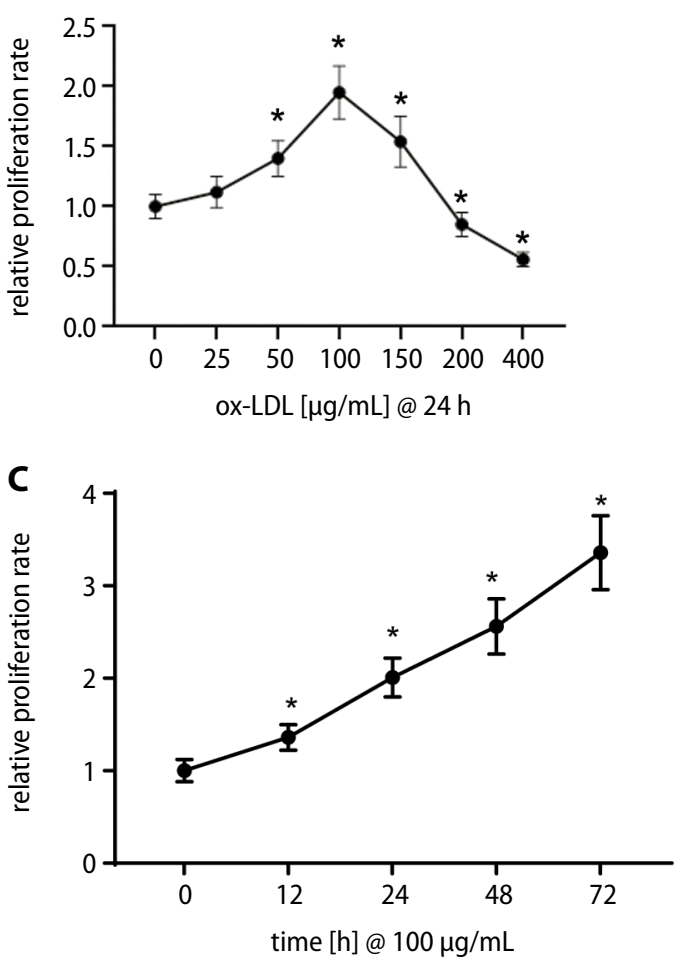

E

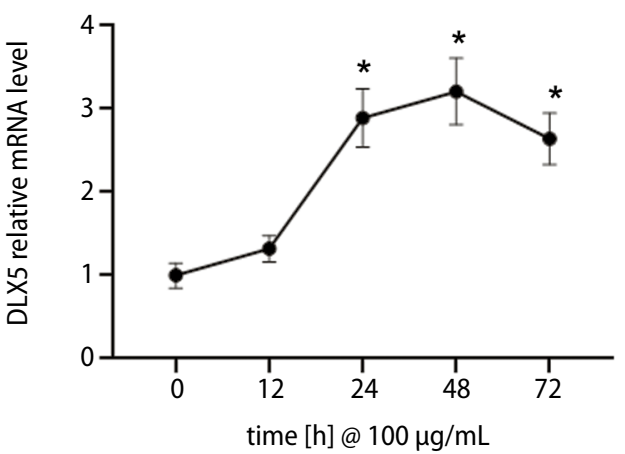

B

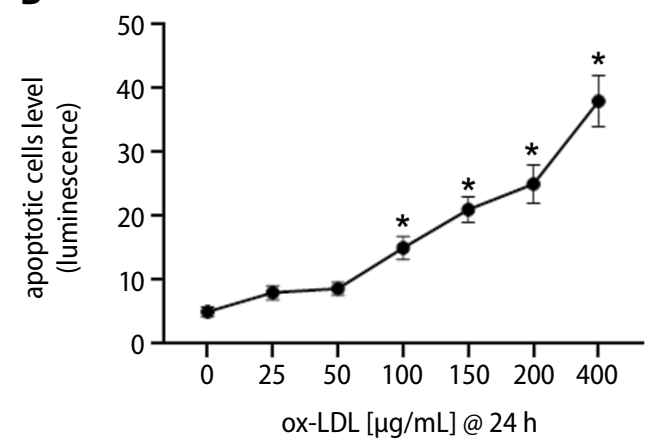

D

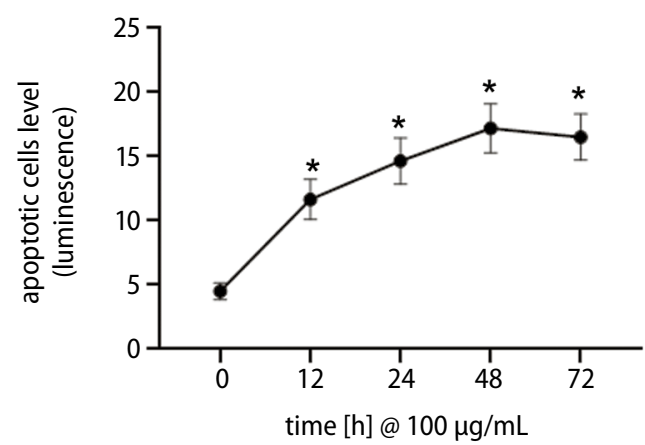

$\mathbf{F}$

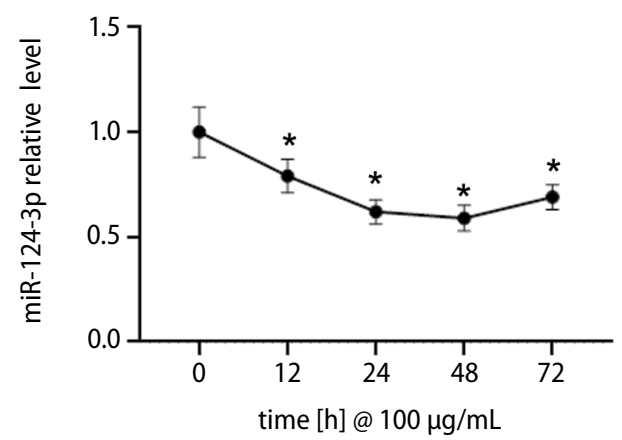

Fig. 5. Dose- and time-dependent effect of oxidized lowdensity lipoprotein (ox-LDL) on DLX5.

$A$ and B. The vascular smooth muscle cells (VSMCs) were treated with different concentration of ox-LDL. Proliferation and apoptosis were analyzed;

$C$ and D. The VSMCs were treated with $100 \mu \mathrm{g} / \mathrm{mL}$ of ox-LDL for different timepoints. Proliferation and apoptosis were analyzed; $E$ and F. The DLX5 and miR-124-3p were detected in $100 \mu \mathrm{g} / \mathrm{mL}$ of VSMCs treatment for different timepoints

* $p<0.05$ compared with control; n=3; @ - indicated time point or concentration.
(Fig. 7A). The $D L X 5$ had a stimulatory effect on VSMCs proliferation ( $\mathrm{p}=0.003)$, while siRNA-DLX5 inhibited proliferation $(\mathrm{p}=0.016)$ (Fig. $7 \mathrm{~B})$. According to our flow cytometry results, transfection with miR-124-3p mimics and siRNA- $D L X 5$ arrested the cell cycle at the $\mathrm{G}_{0} / \mathrm{G}_{1}$ phase ( $p=0.02$ and 0.03 , respectively) (Fig. 7C,D), and induced apoptosis ( $\mathrm{p}=0.005$ and 0.008 , respectively) (Fig. 7E,F). However, transfection with miR-124-3p inhibitor and pcDNA3.1-DLX5 increased the proportion of cells in G2/M phases, but with no effect on apoptosis. When investigating proliferation, cell cycle and apoptosis of VSMCs, overexpression of DLX5 antagonized the effect of miR-124-3p mimics.

Following transfection with miR-124-3p mimics and siRNA-DLX5 for $48 \mathrm{~h}$, cells were treated with ox-LDL for $24 \mathrm{~h}$. As expected, ox-LDL treatment significantly increased VSMC proliferation ( $\mathrm{p}<0.05)$ (Fig. 8A) and increased the proportion of cells in $S$ and $G_{2} / M$ phases $(p=0.03)$ (Fig. 8B). The overexpression of miR-124-3p or inhibition of $D L X 5$ antagonized the effect of ox-LDL significantly ( $\mathrm{p}=0.03$ and $\mathrm{p}=0.004$, respectively). The oxLDL tended to induce VSMC apoptosis, and this effect was enhanced by upregulation of miR-124-3p or inhibition of DLX5 (Fig. 8C). Together, ox-LDL regulates the VSMC proliferation/apoptosis balance through the miR-124-3p/ $D L X 5$ axis.

\section{Discussion}

Recent studies have shown that ox-LDL may regulate the VSMC proliferation/apoptosis balance through miRs, making ox-LDL-related miR pathways promising therapeutic targets for AS treatment. ${ }^{12}$ In this study, we determined mRNA and miR expression in VSMCs $24 \mathrm{~h}$ after ox-LDL treatment using high-throughput RNA sequencing. These data demonstrated significant changes to both, mRNA and miR expressions following ox-LDL treatment. 
A

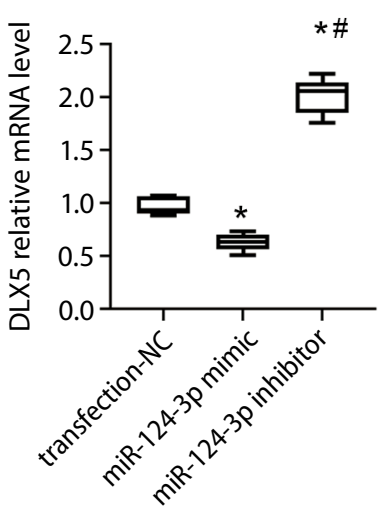

D

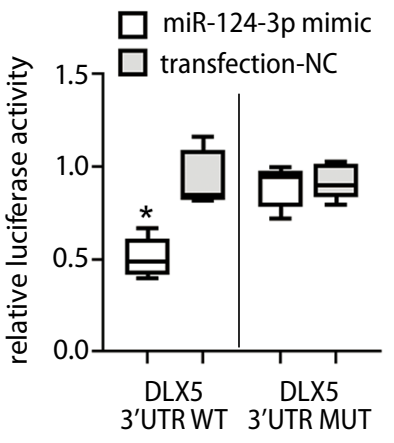

B

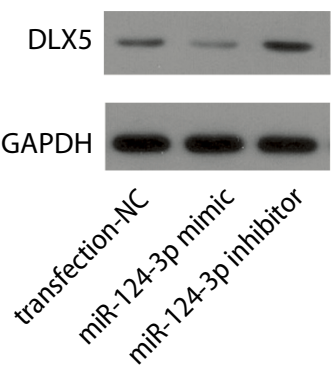

C

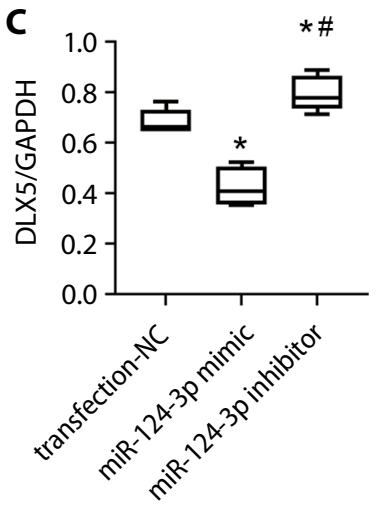

Fig. 6. The miR-124-3p negatively regulated $D L X 5$ expression. A. Up- or downregulation of miR-124$3 p$ changed the mRNA level of $D L X 5$; $B$ and C. Up- or downregulation of miR-124-3p changed the protein level of DLX5; D. The miR-124-3p binding to the DLX5 3'UTR was indicated using luciferase reporter assay (left). The wild-type and mutant DLX5 3'UTR sequence and the predicted binding site to miR-124$3 p$ is shown (right)

* $p<0.05$ compared with transfection negative control (NC); ${ }^{*} p<0.05$ compared with miR-124-3p mimic.

\author{
DLX5 3' UTR WT \\ 5'-caCUUUCAAUG - GUGCCUUg-3' \\ miR-124-3p \\ 3'-ccGUAAGUGGCGCACGGAAu-'5 \\ DLX5 3' UTR MUT 5'-caCUUUCAAUG-GUGGGAAg-3'
}

Then, to further reveal the biological functions of these mRNAs and miRs, we conducted functional enrichment analysis of these DE-mRNAs using the GO database, finding that some of the DE-mRNAs are related to the proliferation and cell cycle of VSMCs. In addition, KEGG signal pathway enrichment analysis showed that some of the DEmRNAs are the components of signaling pathways involved in VSMC functions. The DIANA-mirPath (http:// snf-515788.vm.okeanos.grnet.gr/) is an online tool that reveals the function of miRs of interest through pathway enrichment analysis of target genes for those miRs. This analysis highlighted that cell cycle, apoptosis and MAPK and RAS signaling pathways are involved after ox-LDL treatment. This evidence indicated that miRs might also participate in ox-LDL-induced VSMC proliferation/apoptosis imbalance.

Because it was not possible for us to verify all these sequencing data, it was necessary to screen out mRNAs of interest and their corresponding miRs for further analysis. With a focus on the regulatory effect of ox-LDL on the VSMC proliferation/apoptosis balance, together with the results of GO gene enrichment analysis, only DEgenes related to the proliferation and cell cycle regulation of VSMCs were selected as candidates for verification. However, we found many such candidates, and we selected the gene with the highest expression of FC, $D L X 5$, for further analysis. Next, miRs that can use $D L X 5$ as the target gene were predicted and compared with previously obtained DEmiRs. These candidate miRs should be expressed at a low level in ox-LDL-treated VSMCs and bound to the 3'UTR of $D L X 5$. Finally, we only selected miR-124-3p in this study and assumed that ox-LDL can regulate the VSMC proliferation/apoptosis balance through the miR-124-3p/DLX5 axis, which was verified in vitro. Quantitative real-time polymerase chain reaction (RT-qPCR) confirmed that miR124-3p expression decreased in ox-LDL-treated VSMCs. In addition, the expression of mRNA and protein levels of DLX5 increased in VSMCs after ox-LDL treatment. Furthermore, we confirmed the targeted inhibitory effect of miR-124-3p on DLX5. Together, these data indicated the presence of the miR-124-3p/DLX5 axis that can be regulated by ox-LDL in VSMCs.

Some studies investigating cancer have reported that miR-124-3p inhibits cell proliferation. Xu et al. showed that miR-124-3p inhibits the proliferation of nasopharyngeal carcinoma cells, ${ }^{13}$ while $\mathrm{Wu}$ et al. reported the inhibitory effect of miR-124-3p on glioma proliferation. ${ }^{14}$ The inhibitory effect of miR-124-3p on VSMC proliferation and migration has also been reported previously. ${ }^{15}$ However, Cheng et al. showed that miR-124-3p promotes VSMC proliferation, so the role of miR-124-3p in VSMCs remains controversial. ${ }^{16}$ The current study showed that the upregulation of miR-124-3p inhibits VSMC proliferation, arrests the cell cycle and increases apoptosis, while its inhibition accelerates VSMC proliferation. Therefore, we believe that miR-124-3p can inhibit VSMC growth. Moreover, we discovered that miR-124-3p overexpression counteracts the stimulatory effect of ox-LDL on VSMC proliferation. 

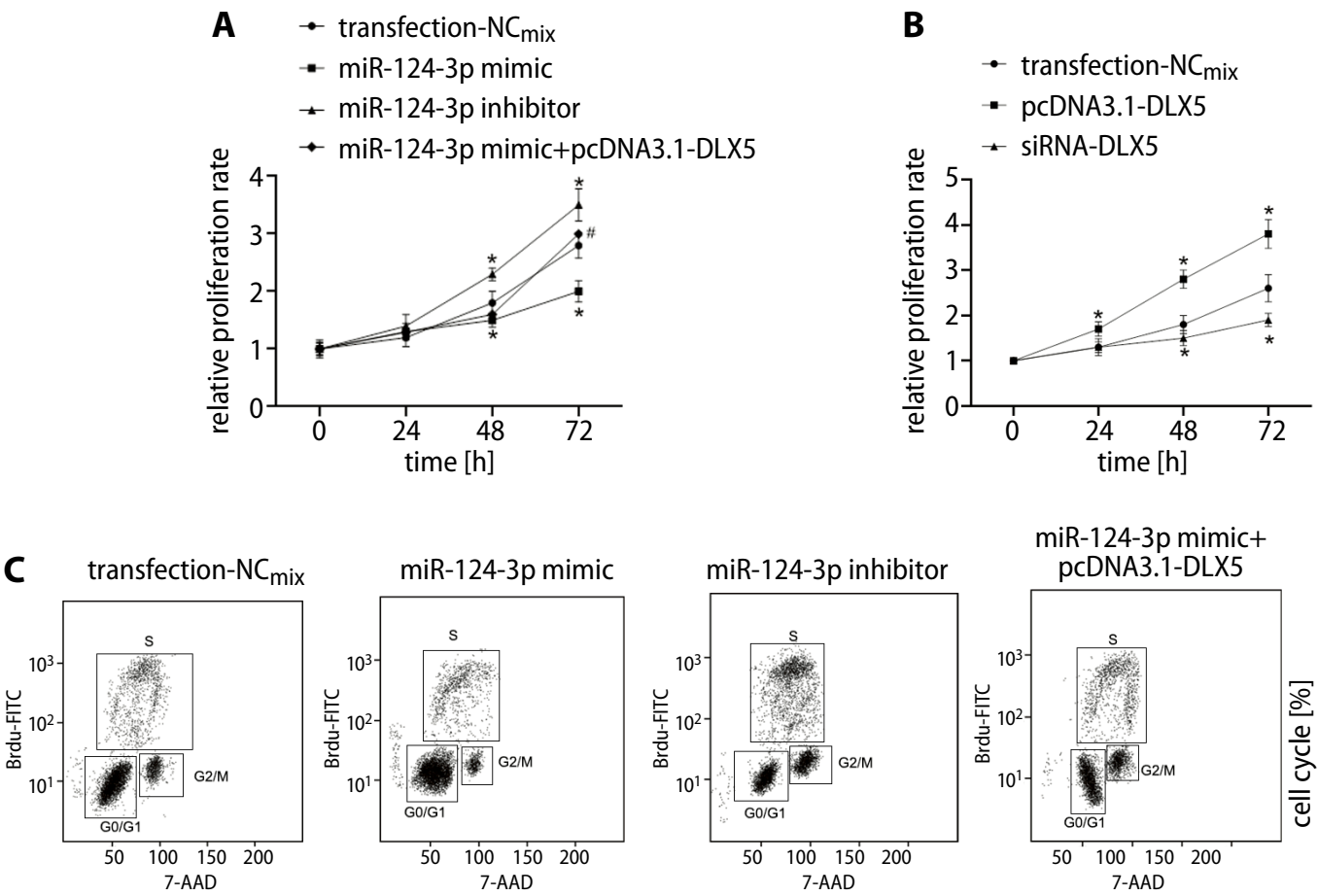

๑transfection-NC amiR-124-3p mimic amiR-124-3p inhibitor amiR-124-3p mimic+ pCDNA3.1-DLX5
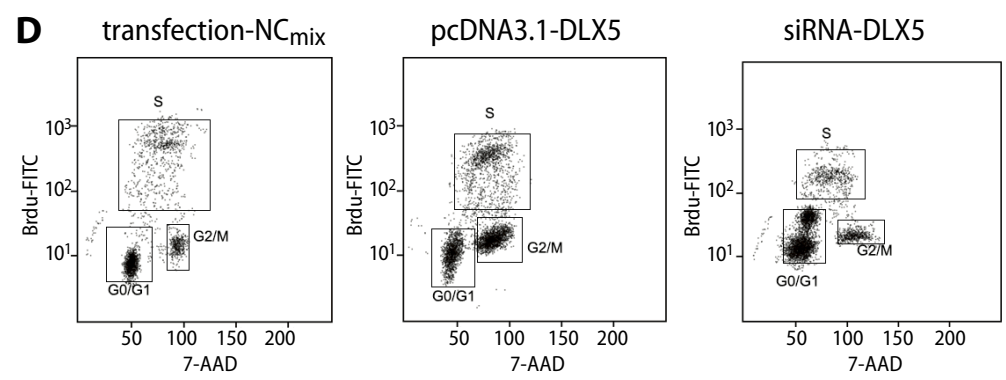

\section{$\square$ transfection-NC pCDNA3.1-DLX5 $\square$ SiRNA-DLX5}

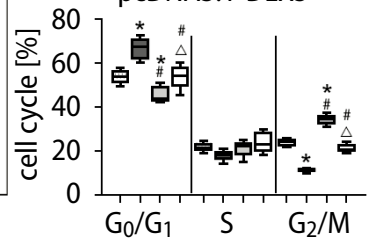
E $\quad$ transfection-NC
$\square$ miR-124-3p mimic
$\square$ miR-124-3p inhibitor
$\square$ miR-124-3p mimic+pcDNA3.1-DLX5
F $\quad$ 圖 transfection-NC
$\square$ pcDNA3.1-DLX5
$\square$ siRNA-DLX5
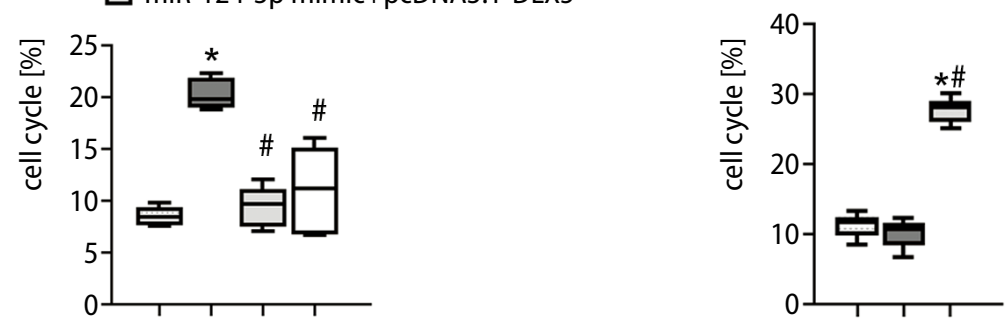

Fig. 7. Functional roles of miR-124-3p and DLX5 on proliferation, cell cycle and apoptosis in human aorta vascular smooth muscle cells (HA-VSMCs). $A$ and B. The miR-124-3p inhibited proliferation of HA-VSMCs, but DLX5 promoted cell proliferation; $C$ and D. The miR-124-3p caused $G_{1} / G_{0}$ phase arrest, while $D L X 5$ increased proportion of $\mathrm{G}_{2} / \mathrm{M}$; $E$ and F. Upregulation of miR-124-3p and knockdown of $D L X 5$ induced cells apoptosis

${ }^{*} p<0.05$ compared with transfection negative control (NC) mixture; $p<0.05$ compared with miR-124-3p mimic or $p c D N A 3.1-D L X 5 ;{ }^{\triangle} p<0.05$ compared with miR-124-3p inhibitor. Transfection $N C_{\text {mix }}$ was a mixture of Allstars sequence, miScript Inhibitor Negative Control and empty pcDNA3.1 in A, C and E. Transfection NC ${ }^{\text {mix }}$ was a mixture of pcDNA3.1 empty vectors and Allstars sequence in $B, D$ and $F(n=5)$.

These results show that ox-LDL induces VSMC proliferation partially by regulating miR-124-3p.

One of the target genes of miR-124-3p, DLX5, was further investigated to explore its underlying mechanisms. The DLX5 is a member of the protein family encoded by DLX (Drosophila distal-less) homeobox genes, and a transcription factor. ${ }^{17}$ Recent studies have shown that
DLX5 promotes cell proliferation by regulating the MYC and protein kinase B (AKT) pathways. ${ }^{18}$ Tan et al. found that $D L X 5$ knockdown inhibits the proliferation of ovarian cancer cells and arrests the cell cycle at the $G_{0} / G_{1}$ phase. ${ }^{19}$ Furthermore, previous results on $D L X 5$ functions were consistent with our results. Previous evidence, supporting the promoting effect of $D L X 5$ on cell proliferation, 
A

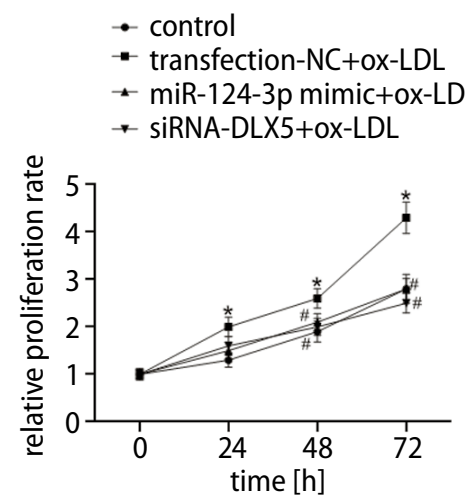
C $\quad$ ontrol $\square$ transfection-NC $\square$ miR-124-3p mimic siRNA-DLX5

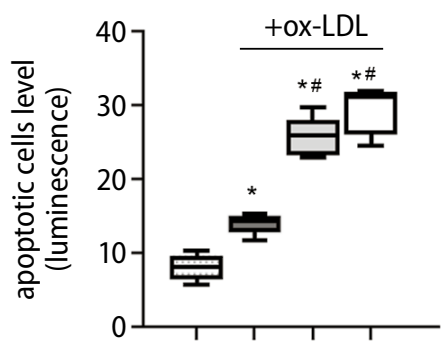

B
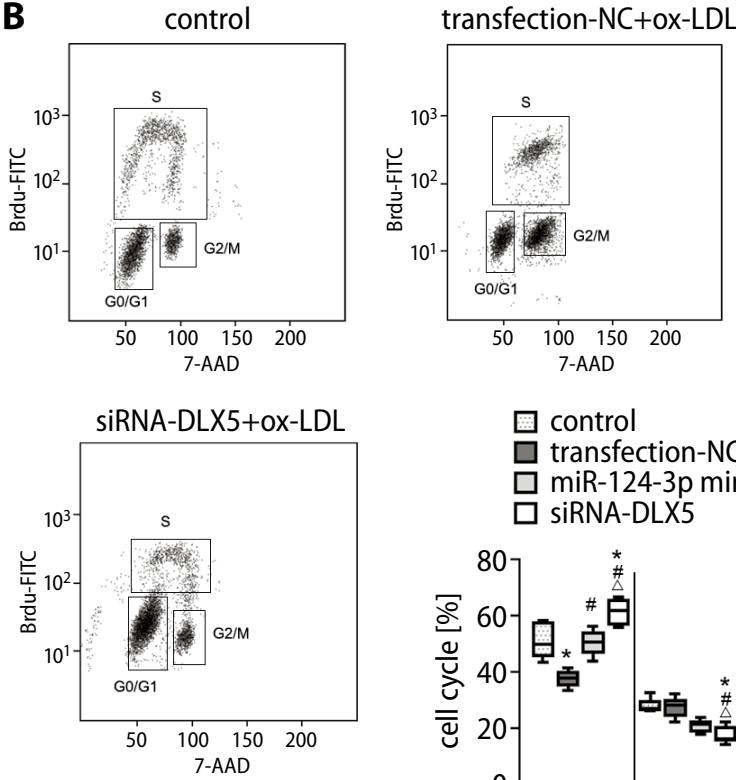

$\square$ control

transfection-NC
miR-124-3p mimic+ox-LDL

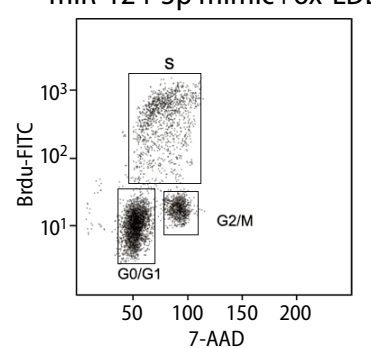
\begin{tabular}{l|l}
$\square$ miR-124-3p mimic & +ox-LDL
\end{tabular}

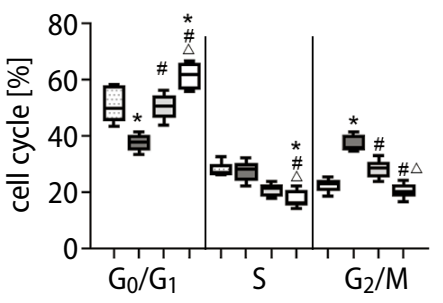

Fig. 8. Oxidized low-density lipoprotein (ox-LDL) regulates the proliferation/apoptosis balance $F(n=5)$. The miR-124-3p/DLX5 axis in human aorta vascular smooth muscle cells (HA-VSMCs). A. The ox-LDL induced proliferation of HA-VSMCs. Upregulation of miR-124-3p or knockdown of $D L X 5$ antagonized this effect; $B$. Upregulation of miR-124-3p or knockdown of $D L X 5$ counteracted the increased $G_{2} / M$ phase induced by ox-LDL; $C$. Upregulation of miR124-3p or knockdown of DLX5 enhanced apoptosis-promoting effect of ox-LDL

${ }^{*} p<0.05$ compared with control; \# $p<0.05$ compared with transfection negative control (NC); ${ }^{\Delta} p<0.05$ compared with miR-124-3p mimic.

is mainly derived from tumor studies, and the role of $D L X 5$ in VSMCs has limited literature. Our results showed that $D L X 5$ overexpression increases VSMC proliferation, while $D L X 5$ knockdown inhibits this process, arrests the cell cycle and increases apoptosis. Therefore, we believe that the function of DLX5 in VSMCs is consistent with that in tumor cells (i.e., promotion of cell proliferation). Although we verified the function of the miR-124-3p/DLX5 axis in VSMCs, we did not explore related mechanisms any further. Future studies may examine the target gene of $D L X 5$ to shed light on any potential downstream effects. In addition, the miR-124-3p/DLX5 axis was selected due to the expression FC, and the functions of other miRmRNA axes that play a role in ox-LDL-treated VSMCs were ignored, which was also a limitation of this study.

\section{Limitations}

We did not explain how ox-LDL inhibits miR-124-3p levels. Whether ox-LDL reduces miR-124-3p by affecting transcription or by posttranscriptional regulation needs to be confirmed in subsequent studies.

\section{Conclusion}

In summary, ox-LDL affects the VSMC proliferation/ apoptosis balance through the miR-124-3p/DLX5 axis and eventually results in VSMC hyperproliferation. The miR124-3p/DLX5 axis might serve as a therapeutic molecular target to reverse the effect of ox-LDL and prevent AS development and progression.

\section{Availability of data and materials}

The RNA-seq data generated and analyzed during the current study are available in the Sequence Read Archive (SRA) database in National Center for Biotechnology Information (NCBI). Data can be accessed at https://www.ncbi.nlm.nih. gov/bioproject/PRJNA714763.

\section{ORCID iDs}

Chunwen Jia (1) https://orcid.org/0000-0002-6578-9554 Feng Gao (i) https://orcid.org/0000-0002-2695-1280 Yanan Zhao (1) https://orcid.org/0000-0002-4854-5689 Siyang Ji (D) https://orcid.org/0000-0003-2137-5314 Shidao Cai (1) https://orcid.org/0000-0003-3116-5863 


\section{References}

1. Kobiyama K, Ley K. Atherosclerosis. Circ Res. 2018;123(10):1118-1120. doi:10.1161/CIRCRESAHA.118.313816

2. Gimbrone MA, García-Cardeña G. Endothelial cell dysfunction and the pathobiology of atherosclerosis. Circ Res. 2016;118(4):620-636. doi:10.1161/CIRCRESAHA.115.306301

3. Dzau VJ, Braun-Dullaeus RC, Sedding DG. Vascular proliferation and atherosclerosis: New perspectives and therapeutic strategies. NatMed. 2002;8(11):1249-1256. doi:10.1038/nm1102-1249

4. Kattoor AJ, Kanuri SH, Mehta JL. Role of Ox-LDL and LOX-1 in atherogenesis. Curr Med Chem. 2019;26(9):1693-1700. doi:10.2174/092986 7325666180508100950

5. Gao S, Zhao D, Wang M, et al. Association between circulating oxidized LDL and atherosclerotic cardiovascular disease: A meta-analysis of observational studies. Can J Cardiol. 2017;33(12):1624-1632. doi:10.1016/j.cjca.2017.07.015

6. Mitra S, Goyal T, Mehta JL. Oxidized LDL, LOX-1 and atherosclerosis. Cardiovasc Drugs Ther. 2011;25(5):419-429. doi:10.1007/s10557-0116341-5

7. Pirillo A, Norata GD, Catapano AL. LOX-1, OxLDL, and atherosclerosis Mediators Inflamm. 2013;2013:152786. doi:10.1155/2013/152786

8. Rhoads JP, Major AS. How oxidized low-density lipoprotein activates inflammatory responses. Crit Rev Immunol. 2018;38(4):333-342. doi:10.1615/CritRevImmunol.2018026483

9. Balzan S, Lubrano V. LOX-1 receptor: A potential link in atherosclerosis and cancer. Life Sci. 2018;198:79-86. doi:10.1016/j.Ifs.2018.02.024

10. Sun Y, Chen D, Cao L, et al. MiR-490-3p modulates the proliferation of vascular smooth muscle cells induced by ox-LDL through targeting PAPP-A. Cardiovasc Res. 2013;100(2):272-279. doi:10.1093/cvr/cvt172

11. Wang YS, Wang HYJ, Liao YC, et al. MicroRNA-195 regulates vascular smooth muscle cell phenotype and prevents neointimal formation. Cardiovasc Res. 2012;95(4):517-526. doi:10.1093/cvr/cvs223
12. Nilsson J, Nordin Fredrikson G, Schiopu A, Shah PK, Jansson B, Carlsson R. Oxidized LDL antibodies in treatment and risk assessment of atherosclerosis and associated cardiovascular disease. Curr Pharm Des. 2007; 13(10):1021-1030. doi:10.2174/138161207780487557

13. Xu S, Zhao N, Hui L, Song M, Miao ZW, Jiang XJ. MicroRNA-124-3p inhibits the growth and metastasis of nasopharyngeal carcinoma cells by targeting STAT3. Oncol Rep. 2016;35(3):1385-1394. doi:10. 3892/or.2015.4524

14. Wu Q, Xu L, Wang C, Fan W, Yan H, Li Q. MicroRNA-124-3p represses cell growth and cell motility by targeting EphA2 in glioma. Biochem Biophys Res Commun. 2018;503(4):2436-2442. doi:10.1016/j. bbrc.2018.06.173

15. Afzal TA, Luong LA, Chen D, et al. NCK Associated protein 1 modulated by miRNA-214 determines vascular smooth muscle cell migration, proliferation, and neointima hyperplasia. J Am Heart Assoc. 2016; 5(12):e004629. doi:10.1161/JAHA.116.004629

16. Cheng C, Xu BL, Sheng JL, He F, Yang T, Shen SC. LncRNA MALAT1 regulates proliferation and apoptosis of vascular smooth muscle cells by targeting miRNA-124-3p/PPARa axis. Eur Rev Med Pharmacol Sci. 2019;23(20):9025-9032. doi:10.26355/eurrev_201910_19304

17. Eisenstat DD, Liu JK, Mione $M$, et al. DLX-1, DLX-2, and DLX-5 expression define distinct stages of basal forebrain differentiation. J Comp Neurol. 1999;414(2):217-237. doi:10.1002/(sici)1096-9861(1999 1115) 414:2<217::aid-cne6>3.0.co;2-i

18. Tan $Y$, Sementino $E, X u J$, et al. The homeoprotein D/x5 drives murine T-cell lymphomagenesis by directly transactivating Notch and upregulating Akt signaling. Oncotarget. 2017;8(9):14941-14956. doi:10.18632/oncotarget.14784

19. Tan Y, Cheung M, Pei J, Menges CW, Godwin AK, Testa JR. Upregulation of $D L X 5$ promotes ovarian cancer cell proliferation by enhancing IRS-2-AKT signaling. Cancer Res. 2010;70(22):9197-9206. doi:10.1158/ 0008-5472.CAN-10-1568 